\title{
Economic Outcomes of First-Line Regimen Switching Among Stable Patients with HIV
}

\author{
Lisa Rosenblatt, MD, MPH; Ami R. Buikema, MPH; Jerry Seare, MD; \\ Lindsay G. S. Bengtson, PhD, MPH; Jonathan Johnson, MS; Feng Cao, PhD; \\ and Angelina Villasis-Keever, MD, MSc
}

\begin{abstract}
BACKGROUND: Although switching of antiretroviral therapy (ART) is a valid approach for addressing treatment failure in patients with human immunodeficiency virus (HIV), ART changes among those who are well maintained on their current regimens may lead to the development of new side effects or resistance.
\end{abstract}

OBJECTIVE: To examine the effect of first-line regimen switching on subsequent health care utilization and cost among stable HIV patients.

METHODS: This was a retrospective claims data study of adult patients with HIV who initiated ART between 2007 and 2013 and had been treated with their initial regimens for at least 6 continuous months. Those with evidence of pregnancy or HIV-2 were excluded. Patients who underwent an ART change were assigned to a switcher cohort; a nonswitcher cohort was then generated by matching up to 20 nonswitchers for each switcher, with replacement. The index date was the date of the first ART change for switchers and was the claim date closest to the corresponding switcher's switch date for nonswitchers. Patient characteristics at baseline and postindex annualized health care utilization and costs were analyzed descriptively and with multivariable models. Analyses were performed in the full population and among patients designated as virologically stable (had undetectable viral ribonucleic acid [RNA] for 90 days pre-index) and virologically and clinically stable (had undetectable viral RNA and no apparent clinical reason for switching ART).

RESULTS: The study population consisted of 6,983 individuals, which included 927 switchers (168 virologically stable; 55 virologically+clinically stable), who were matched with replacement with 18,511 nonswitcher comparators. The switcher cohort was $88.8 \%$ male (mean age 43.8 years). Mean preindex and follow-up treatment durations for switchers and nonswitchers were 1.8 years and 1.5 years, respectively; demographic characteristics, pre-index treatment duration, and follow-up duration were similar between cohorts. Significantly more nonswitchers than switchers had a first-line efavirenz-based regimen $(67.1 \%$ vs. $47.8 \%, P<0.001)$. In the virologically stable subset, follow-up annualized health care utilization for switchers versus nonswitchers, respectively, was 14.8 versus 12.3 ambulatory visits $(P<0.05), 0.8$ versus 0.9 emergency department visits $(P=0.652)$, and 0.05 versus 0.05 inpatient hospitalizations $(P=0.915)$. Follow-up annualized health care costs were $\$ 37,120$ for switchers versus $\$ 31,771$ for nonswitchers $(P<0.05)$, with the difference driven largely by pharmacy costs. Multivariable-adjusted follow-up annualized health care costs were $8.9 \%$ higher among switchers versus nonswitchers $(P<0.01)$, and switchers also had a shorter time to subsequent ART regimen change $(P<0.001)$. Results were similar for the virologically+clinically stable subset.

CONCLUSIONS: In this large, real-world population, stable patients with HIV who switched from their first-line ART regimens had significantly higher health care costs than those who did not change therapies, suggesting that ART regimen changes may be costly and should be undertaken only when clinically warranted.

\section{J Manag Care Spec Pharm. 2017;23(7):725-34}

Copyright $\odot 2017$, Academy of Managed Care Pharmacy. All rights reserved.

\section{What is already known about this subject}

Modern antiretroviral therapy (ART) now allows patients with human immunodeficiency virus (HIV) infection to live a nearnormal lifespan, but despite substantial improvements in the convenience and tolerability of ART regimens, challenges related to medication adherence and quality of life remain for some patients.

ART regimen switching may be needed in patients with virologic failure to address antiviral resistance or in virally suppressed patients to manage drug toxicity, improve adherence, or reduce costs; however, regimen changes also carry the risk of new adverse events, drug interactions, or loss of virologic suppression. Some real-world studies have found that health care costs among patients with HIV increased after a switch to a secondor third-line regimen, but these studies were not confined to stable patients and were conducted before some currently recommended therapies were on the market.

\section{What this study adds}

This study examined the effect of ART regimen switching on health care utilization and costs among stable HIV patients.

- Among virologically stable patients with HIV, adjusted followup health care costs were $8.9 \%$ higher for those who switched regimens compared with those remaining on their first-line regimens, an increase driven almost exclusively by higher HIVrelated pharmacy costs

Time to next ART regimen change was shorter for switchers compared with nonswitchers; although this could suggest decreased stability of virologic suppression, it is possible that patients who had already switched regimens were more open to considering a subsequent switch.

A dvances in antiretroviral therapy (ART) have rendered human immunodeficiency virus (HIV) infection a chronic disease; with effective treatment, individuals with HIV can now live a near-normal lifespan. ${ }^{1}$ Patients are generally initiated on a regimen that includes 2 nucleoside reverse transcriptase inhibitors (NRTIs) and 1 agent from another class of antiretroviral drugs (either a protease inhibitor [PI] or an integrase strand transfer inhibitor, according to 
current guidelines). ${ }^{2}$ While highly effective, the drugs used in ART regimens may cause significant side effects, including rashes, insomnia, liver toxicity, renal or bone disease, gastrointestinal intolerance, lipid abnormalities, and increased cardiovascular disease risk, which must be considered in conjunction with patient history and comorbid conditions when choosing a treatment regimen. ${ }^{2}$ Adverse effects of ART can diminish quality of life and reduce adherence, ${ }^{3,4}$ which is a critical issue; because there is no cure for HIV infection, high lifetime ART adherence to achieve and maintain virologic suppression is requisite. $^{5}$

ART regimen switching is a common approach for addressing virologic failure, ${ }^{2,6}$ but switching is also done in patients with sustained viral suppression to manage drug toxicity and interactions, improve adherence, or reduce costs. ${ }^{2}$ There is ample evidence that switching ART in virologically stable patients-for example, from boosted PIs to non-nucleoside reverse transcriptase inhibitors-can improve quality of life, reduce adverse effects, and improve metabolic profile while maintaining virologic control ${ }^{3,7-13}$; however, ART switches can also be associated with new adverse events and even lead to worse clinical outcomes. ${ }^{14,15}$ Moreover, treatment changes carry the possibility of more frequent office visits and overlapping prescriptions, which could increase health care costs. The decision to switch ART regimens thus requires careful consideration and must be personalized to the medical and social history of each individual patient.

The results of many clinical trials examining the effect of ART switching are limited because they were conducted before currently recommended therapies were commercially available and were concerned primarily with metrics such as virologic suppression, adherence, and quality of life rather than economic assessment.,12,16,17 More recent real-world studies have found that health care costs among patients with HIV increased after a switch to a second- or third-line regimen. . $^{18,19}$ However, since these studies were not confined to stable patients, their results may reflect the higher costs of treating those with poorly controlled disease. Few recent studies have examined the effect of stable ART switching on subsequent health care utilization and costs. ${ }^{20}$ We thus sought to quantify the health care burden associated with therapy changes among HIV-positive patients who have longevity on their first-line ART regimens.

\section{Methods}

\section{Data Sources}

This was a retrospective study using administrative claims data from the Optum Research Database and the Impact National Benchmark Database. These databases combined include enrollment information, medical and pharmacy claims, and laboratory test results for approximately 51.8 million individuals with commercial medical and pharmacy benefit coverage and at least 1 day of enrollment between January 1, 2007, and December 31, 2013. The databases comprise a geographically diverse and representative U.S. commercially insured population. Medical claims include diagnosis, procedure, revenue, and site of service codes. Outpatient pharmacy claims include medications dispensed, with quantity, dose, and number of days supply. Laboratory test results are available for 30\%-40\% of patients in the databases and include Logical Observation Identifiers Names and Codes, vendorspecific test codes, and result values. Since no protected health information was extracted or accessed during the course of this study, approval by an institutional review board or waiver of authorization was not required.

\section{Study Sample Selection and Cohort Assignment}

The study population consisted of commercial health plan enrollees initiating ART treatment between January 1, 2007, and December 31, 2013. The treatment initiation date was defined as the date of the first ART prescription fill preceded by a "clean" period of at least 6 months with no evidence of ART. The first-line ART regimen included all ART prescriptions filled within 14 days of the treatment initiation date. To be eligible, patients must have initiated an ART regimen of 3 or more drugs that was recommended in the current U.S. Department of Health and Human Services treatment guidelines for HIV infection. $^{2}$ Regimens included in previous versions of the guidelines at any time during the study period were also allowed. To exclude early switches that were more likely to be related to tolerability, patients must have been treated with the first-line regimen for at least 6 continuous months with no therapy changes. Additional inclusion requirements were age 18 years or older; HIV diagnosis (International Classification of Diseases, Ninth Revision, Clinical Modification diagnosis codes 042, 795.71, or V08) at any point during the study period; and complete demographic data (age, sex, and geographic region). Patients with evidence of pregnancy or HIV type 2 were excluded. Patient observation began on the treatment initiation date and lasted until discontinuation of ART (defined as a gap in therapy of more than 90 days), disenrollment from the health plan, or June 30, 2014, whichever occurred first.

Patients who met the selection criteria were assigned to 2 possible cohorts: a switcher cohort and a comparator cohort. Switchers included those who underwent a change in therapy-defined as a prescription fill for an anchor agent or NRTI backbone agent not included in the initial regimenafter at least 6 months of treatment with their first-line ART regimen. For patients in the switcher cohort, the index date was the date of the first ART switch. All patients were eligible for inclusion in the comparator cohort from 6 months after the treatment initiation date up to the date of the first switch (for 
switchers) or the end of patient observation (for patients without an observed switch). Up to 20 comparators of the same sex and initiation year were randomly selected, with replacement, for each switcher. For the comparator cohort, the index date was the closest claim date within 90 days of the corresponding switcher's switch date. The baseline period was defined as the 6 months before the index date. The follow-up period began on the index date and ended on the last day of patient observation. Patients in both cohorts were required to have at least 30 days of post-index follow-up.

Two comparator matches were performed: one each for the full population and the virologically stable subset. Nonswitcher comparators for the virologically stable subset had the added requirement of undetectable HCV ribonucleic acid (RNA) in the 90 days before the index date.

\section{Identification of Stable Patient Subsets}

For the subset analyses, patients in the switcher and nonswitcher comparator cohorts who had an HIV RNA viral load test result in the 90 days before the index date were first identified and classified as virologically stable or unstable according to their HIV RNA status. Those with detectable HIV RNA were considered virologically unstable and excluded from subsequent analysis. The remaining patients were included in the virologically stable subset. The claims histories of the switchers in this subset (from 90 days before the index date through 30 days after the index date) were then subjected to a detailed review by authors AB, LB, JS, LR, and AVK to identify individuals with apparent clinical reasons for switching (Appendix A, available in online article). The information provided for this review included initial treatment regimen, age at treatment initiation, time on initial treatment regimen, pre-index cost trend, index year, switch regimen, diagnosis codes, procedure codes, prescription fills, and laboratory test results. Site of service and number of days from the index date were also provided for each claim. Patients for whom there was evidence that a therapy switch had occurred out of medical necessity (e.g., adverse event diagnosis codes or laboratory test abnormalities) were categorized as clinically unstable and excluded from subsequent analysis; the remaining patients were included in the virologically + clinically stable subset (Figure 1).

\section{Study Measures}

Demographic and Clinical Characteristics. The pretreatment Charlson comorbidity score was calculated on the basis of diagnosis codes from medical claims during the 6-month (minimum) clean period before the treatment initiation date. ${ }^{21}$ The pre-index treatment duration was calculated as the number of days between treatment initiation and the index date. Characteristics assessed during the baseline period were age at index date, sex, geographic region, data source, first-line
ART regimen, comorbidity based on the Charlson Comorbidity Index and Clinical Classifications Software from the Agency for Healthcare Research and Quality, ${ }^{22}$ medication use by American Hospital Formulary Service classification, ${ }^{23}$ and health care costs and cost trajectory. Treatment duration following the index date was also measured, and the second-line regimen was captured among patients who switched therapies.

Outcomes. Outcome variables were measured during the follow-up period. The primary outcomes were health care utilization and costs for switchers compared with nonswitchers. The number of ambulatory, emergency department, and inpatient visits and the duration of inpatient stays were captured to reflect health care utilization. Ambulatory visits were counted per provider per day. Health care costs were calculated as the combined actual health plan-paid and patient-paid amounts based on administrative claims from the index date through the end of the follow-up period, adjusted to 2014 U.S. dollars using the annual medical care component of the Consumer Price Index. ${ }^{24}$ Total health care costs were subdivided into pharmacy (all-cause and HIV-related) and medical costs; the medical category was further subdivided into ambulatory, emergency, inpatient, and other costs.

\section{Statistical Analyses}

All study variables were analyzed descriptively. Results were stratified by switcher versus nonswitcher cohorts, and the matched nonswitchers for each switcher were weighted to represent 1 participant. Bivariate comparisons were analyzed by paired t-tests for continuous variables and by chi-square tests for categorical variables. To control for possible confounding of the association between the primary cost outcome and independent variables of interest-including ART regimen, index year, geographic region, sex, age, Charlson comorbidity score, and baseline health care costs-post-index annualized health care costs were analyzed with a multivariable cost model (generalized linear model with a gamma distribution and log link).

Kaplan-Meier analyses were performed to examine times to virologic failure and next regimen change in the switcher and nonswitcher cohorts. In the analysis examining time to next regimen change, data for patients without a second ART change were censored at the end of the observation period. Kaplan-Meier results were compared between cohorts using a product-limit estimate to account for weighting. All other $P$ values were adjusted using a robust (sandwich) variance estimate to account for correlation caused by multiple observations per patient due to matching with replacement. Statistical significance was defined as $P<0.05$. Statistical analyses were performed using SAS version 9.4 (SAS Institute, Cary, NC). $P$ values were not adjusted for multiple comparisons. 


\section{FIGURE 1 Sample Selection and Attrition Flow Diagram}

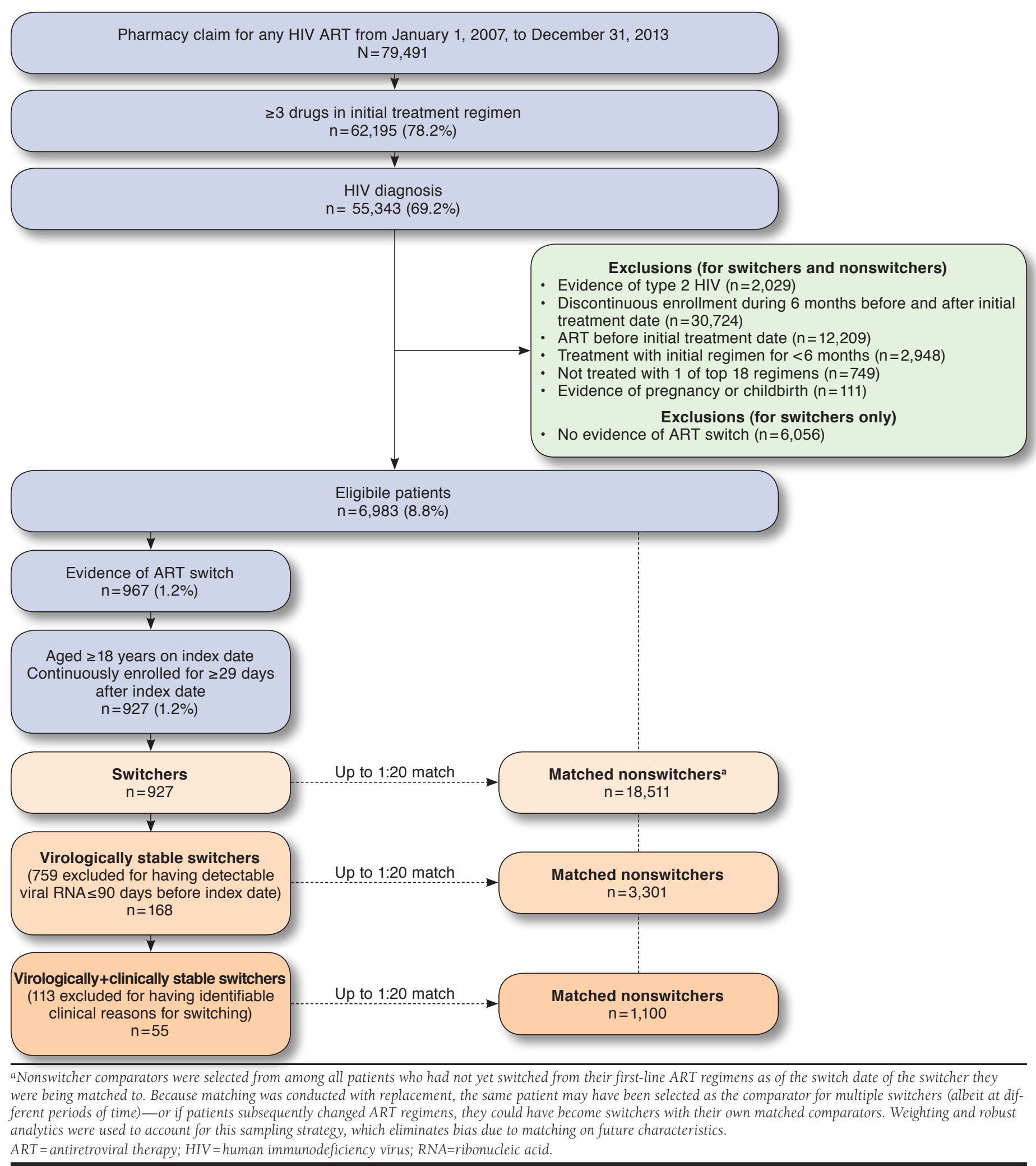


TABLE 1 Baseline Demographic Characteristics

\begin{tabular}{|c|c|c|c|c|c|c|c|c|c|}
\hline \multirow{3}{*}{$\begin{array}{l}\text { Characteristic } \\
\text { Age at index date, mean (SD) }\end{array}$} & \multicolumn{3}{|c|}{ Full Population } & \multicolumn{3}{|c|}{ Virologically Stable } & \multicolumn{3}{|c|}{ Virologically + Clinically Stable } \\
\hline & $\begin{array}{l}\text { Switchers } \\
(\mathbf{n}=927)\end{array}$ & $\begin{array}{c}\text { Nonswitchers } \\
(\mathrm{n}=18,511 \\
\text { weighted } \\
\mathrm{n}=927)\end{array}$ & \multirow{2}{*}{$\frac{P \text { Value }^{\mathrm{b}}}{0.133}$} & $\begin{array}{l}\text { Switchers } \\
(\mathrm{n}=168)\end{array}$ & $\begin{array}{c}\text { Nonswitchers } \\
(\mathbf{n}=3,301 \\
\text { weighted } \\
\mathbf{n}=168)\end{array}$ & \multirow{2}{*}{$\frac{P \text { Value }^{\text {b }}}{0.250}$} & \multirow{2}{*}{$\begin{array}{c}\begin{array}{c}\text { Switchers } \\
(\mathbf{n}=55)\end{array} \\
42.3(10.6)\end{array}$} & $\begin{array}{c}\text { Nonswitchers } \\
(\mathrm{n}=1,100 \\
\text { weighted } \\
\mathrm{n}=55)\end{array}$ & \multirow{2}{*}{$\frac{P \text { Value }^{\mathrm{b}}}{0.673}$} \\
\hline & $43.8 \quad(9.6)$ & $43.3(10.0)$ & & $44.3 \quad(9.6)$ & $43.4(10.0)$ & & & $42.9 \quad(9.7)$ & \\
\hline \multicolumn{10}{|c|}{ Age category at index date, years, $n(\%)^{c}$} \\
\hline $18-44$ & $476(51.4)$ & $493(53.2)$ & 0.272 & $85(50.6)$ & $89(53.2)$ & 0.507 & $33(60.0)$ & $30(55.1)$ & 0.477 \\
\hline $45-64$ & $444(47.9)$ & $421 \quad(45.4)$ & 0.137 & $81(48.2)$ & $76(45.4)$ & 0.482 & $21(38.2)$ & $24(44.2)$ & 0.385 \\
\hline $65+$ & $7 \quad(0.8)$ & $13 \quad(1.4)$ & 0.102 & $2(1.2)$ & $2(1.3)$ & 0.876 & $1 \quad(1.8)$ & $0 \quad(0.7)$ & 0.392 \\
\hline Male sex, $n(\%)^{c}$ & $823(88.8)$ & $823(88.8)$ & - & $146(86.9)$ & $146(86.9)$ & - & $49(89.1)$ & $49(89.1)$ & - \\
\hline \multicolumn{10}{|l|}{ Geographic region, $\mathrm{n}(\%)^{\mathrm{c}}$} \\
\hline Northeast & $262(28.3)$ & $278 \quad(29.9)$ & 0.280 & $22(13.1)$ & $34(20.2)$ & 0.026 & $9(16.4)$ & $12(20.9)$ & 0.423 \\
\hline Midwest & $123(13.3)$ & $125(13.4)$ & 0.880 & $5 \quad(3.0)$ & $8 \quad(4.7)$ & 0.308 & $1 \quad(1.8)$ & $2 \quad(4.4)$ & 0.379 \\
\hline South & $437(47.1)$ & $427(46.1)$ & 0.524 & $108(64.3)$ & $106(63.0)$ & 0.735 & $35(63.6)$ & $33(60.6)$ & 0.659 \\
\hline West & $105(11.3)$ & $98(10.6)$ & 0.452 & $33(19.6)$ & $20(12.1)$ & 0.004 & $10(18.2)$ & $8 \quad(14.1)$ & 0.399 \\
\hline \multicolumn{10}{|l|}{ Index year, $\mathrm{n}(\%)^{\mathrm{c}}$} \\
\hline 2007 & $20 \quad(2.2)$ & $18 \quad(2.0)$ & 0.649 & $1 \quad(0.6)$ & $2 \quad(1.1)$ & 0.562 & \begin{tabular}{ll|}
0 & $(0.0)$ \\
\end{tabular} & $1 \quad(1.2)$ & - \\
\hline 2008 & $84 \quad(9.1)$ & $90 \quad(9.7)$ & 0.525 & $9 \quad(5.4)$ & $10 \quad(5.7)$ & 0.845 & $3 \quad(5.5)$ & $3 \quad(5.5)$ & - \\
\hline 2009 & $116(12.5)$ & $108(11.7)$ & 0.446 & $21(12.5)$ & $20(11.9)$ & 0.799 & $\begin{array}{ll}4 & (7.3) \\
\end{array}$ & $3 \quad(5.5)$ & 0.567 \\
\hline 2010 & $118(12.7)$ & $128(13.9)$ & 0.331 & $23(13.7)$ & $21 \quad(12.3)$ & 0.591 & $7(12.7)$ & $7(12.0)$ & 0.872 \\
\hline 2011 & $131(14.1)$ & $129(13.9)$ & 0.853 & $18(10.7)$ & $17(10.0)$ & 0.771 & $4 \quad(7.3)$ & $4 \quad(6.9)$ & 0.918 \\
\hline 2012 & $173(18.7)$ & $166(18.0)$ & 0.582 & $20(11.9)$ & $25(14.9)$ & 0.292 & $7(12.7)$ & $9(15.8)$ & 0.540 \\
\hline 2013 & $192(20.7)$ & $199(21.5)$ & 0.568 & $42(25.0)$ & $45(26.6)$ & 0.656 & $16(29.1)$ & $20(35.9)$ & 0.308 \\
\hline 2014 & $93(10.0)$ & $87 \quad(9.4)$ & 0.547 & $34(20.2)$ & $30 \quad(17.6)$ & 0.387 & $14(25.5)$ & $10 \quad(17.3)$ & 0.126 \\
\hline \multicolumn{10}{|l|}{ Data source, $\mathrm{n}(\%)^{\mathrm{c}}$} \\
\hline ORD & $657(70.9)$ & $657(70.9)$ & \multirow{2}{*}{0.977} & $154(91.7)$ & $151 \quad(89.9)$ & \multirow{2}{*}{0.457} & $50(90.9)$ & $49(89.9)$ & \multirow{2}{*}{0.812} \\
\hline Impact & $270(29.1)$ & $270 \quad(29.1)$ & & $14 \quad(8.3)$ & $17(10.1)$ & & $\begin{array}{ll}5 & (9.1) \\
\end{array}$ & $6(10.1)$ & \\
\hline \multicolumn{10}{|c|}{$\begin{array}{l}a_{\text {Means, }} \text { SDs, counts, and percentages for nonswitchers are weighted and normalized to the matched switcher cohort. } \\
\text { bP values are adjusted using a robust (sandwich) variance estimate to account for correlation due to multiple observations per patient. } \\
\text { cBecause of rounding, percentages may not sum to } 100 \% \text {. } \\
\text { ORD = Optum Research Database; } S D=\text { standard deviation. }\end{array}$} \\
\hline
\end{tabular}

\section{Results}

\section{Study Sample and Baseline Characteristics}

Of 6,983 eligible patients, 927 (13.3\%) had evidence of an ART switch during the observation period. Among these, 297 (32.0\%) had at least 1 HIV RNA test result available in the 90 days before the index date; 129 of these 297 patients (43.4\%) had detectable viral loads, while 168 (56.6\%) were virally suppressed. Baseline patient demographics and clinical characteristics are shown in Tables 1 and 2. The switcher cohort was $88.8 \%$ male, with a mean age of 43.8 years and a mean pre-index treatment duration of 1.8 years.

Baseline demographic characteristics were similar between switchers and nonswitchers in the full population (Table 1). For baseline clinical characteristics, however, significantly more switchers than nonswitchers had a Charlson comorbidity score of 5 or greater ( $16.9 \%$ vs. $12.9 \%, P<0.001$ ), whereas more nonswitchers had scores of 0 (15.0\% vs. $10.0 \%$, $P<0.001)$ or $1-2(1.1 \%$ vs. $0.3 \%, P=0.040)$. Switchers were also significantly more likely than nonswitchers to have an initial ART regimen with a backbone of atazanavir/ritonavir (14.9\% vs. $7.8 \%, P<0.001)$ or raltegravir ( $8.2 \%$ vs. $5.2 \%, P<0.001)$, whereas more nonswitchers had initiated efavirenz-based regimens (67.1\% vs. $47.8 \%, P<0.001$; Table 2 ).

In the virologically stable and virologically + clinically stable subsets, the Charlson comorbidity score was not significantly different between switchers and nonswitchers; however, other differences in baseline demographic and clinical characteristics were similar to those seen in the full population (Tables 1 and 2).

\section{Health Care Utilization and Costs}

In the full population, follow-up annualized health care utilization per patient for switchers versus nonswitchers was 14.7 versus 11.6 ambulatory visits $(P<0.001), 0.9$ versus 0.6 emergency department visits $(P=0.027)$, and 0.1 versus 0.1 inpatient hospital stays ( $P=0.120$; Table 3$)$. The length of inpatient stays was not statistically different for switchers and nonswitchers (11.2 days vs. 8.7 days, $P=0.485$ ). 


\begin{tabular}{|c|c|c|c|c|c|c|c|c|c|}
\hline \multirow[b]{2}{*}{ Characteristic } & \multicolumn{3}{|c|}{ Full Population } & \multicolumn{3}{|c|}{ Virologically Stable } & \multicolumn{3}{|c|}{ Virologically + Clinically Stable } \\
\hline & $\begin{array}{c}\text { Switchers } \\
(\mathrm{n}=927)\end{array}$ & $\begin{array}{c}\text { Nonswitchers }^{\mathrm{a}} \\
(\mathrm{n}=18,511 \\
\text { weighted } \\
\mathrm{n}=927)\end{array}$ & $P$ Value $^{\mathrm{b}}$ & $\begin{array}{c}\text { Switchers } \\
(\mathrm{n}=168)\end{array}$ & $\begin{array}{c}\text { Nonswitchers } \\
(\mathrm{n}=3,301 \\
\text { weighted } \\
\mathrm{n}=168)\end{array}$ & $P$ Value $^{\mathrm{b}}$ & $\begin{array}{c}\text { Switchers } \\
(\mathrm{n}=55)\end{array}$ & $\begin{array}{c}\text { Nonswitchers } \\
(\mathrm{n}=1,100 \\
\text { weighted } \\
(\mathrm{n}=55)\end{array}$ & $P$ Value $^{\mathrm{b}}$ \\
\hline \multicolumn{10}{|c|}{ Charlson comorbidity score category, $\mathrm{n}(\%)^{\mathrm{c}}$} \\
\hline 0 & $93(10.0)$ & $139(15.0)$ & $<0.001$ & $15 \quad(8.9)$ & $18 \quad(10.9)$ & 0.441 & $10(18.2)$ & $6 \quad(10.2)$ & 0.068 \\
\hline $1-2$ & $3 \quad(0.3)$ & $10 \quad(1.1)$ & 0.040 & $2(1.2)$ & $1 \quad(0.8)$ & 0.634 & $1 \quad(1.8)$ & $1 \quad(1.0)$ & 0.568 \\
\hline $3-4$ & $674(72.7)$ & $658(71.0)$ & 0.267 & $118(70.2)$ & $124 \quad(73.6)$ & 0.339 & $40(72.7)$ & 42 (75.6) & 0.639 \\
\hline $5+$ & $157(16.9)$ & $120(12.9)$ & $<0.001$ & $33(19.6)$ & $25 \quad(14.7)$ & 0.085 & $4 \quad(7.3)$ & $7(13.3)$ & 0.207 \\
\hline $\begin{array}{l}\text { Years of treatment pre-index, } \\
\text { mean (SD) }\end{array}$ & $1.8 \quad(1.2)$ & $1.8 \quad(1.2)$ & 0.962 & $2.0 \quad(1.1)$ & $2.0 \quad(1.1)$ & 0.991 & $1.9 \quad(1.1)$ & $1.9 \quad(1.1)$ & 0.996 \\
\hline \multicolumn{10}{|c|}{ Antiretroviral treatment regimen, ${ }^{\mathrm{d}} \mathrm{n}(\%)^{\mathrm{c}}$} \\
\hline $\mathrm{EFV}+\mathrm{TDF}+\mathrm{FTC}$ & $400(43.2)$ & $603(65.1)$ & $<0.001$ & $71(42.3)$ & $106 \quad(63.2)$ & $<0.001$ & $14(25.5)$ & $34(61.3)$ & $<0.001$ \\
\hline $\mathrm{ATV} / \mathrm{r}+\mathrm{TDF}+\mathrm{FTC}$ & $127(14.9)$ & $63 \quad(7.8)$ & $<0.001$ & $25(14.9)$ & $8 \quad(4.8)$ & $<0.001$ & $12(21.8)$ & $3 \quad(4.9)$ & $<0.001$ \\
\hline RAL+TDF+FTC & $76 \quad(8.2)$ & $48 \quad(5.2)$ & $<0.001$ & $13(7.7)$ & $9 \quad(5.3)$ & 0.178 & $9(16.4)$ & $3 \quad(5.4)$ & 0.001 \\
\hline DRV/r+TDF+FTC & $62 \quad(6.7)$ & $43 \quad(4.7)$ & 0.005 & $\begin{array}{ll}7 & (4.2) \\
\end{array}$ & $10 \quad(5.7)$ & 0.425 & $3 \quad(5.5)$ & $3 \quad(5.6)$ & 0.955 \\
\hline $\mathrm{LPV} / \mathrm{r}+\mathrm{TDF}+\mathrm{FTC}$ & $77 \quad(8.3)$ & $25 \quad(2.7)$ & $<0.001$ & $17(10.1)$ & $(2.4)$ & $<0.001$ & $\begin{array}{ll}5 & (9.1) \\
\end{array}$ & $1 \quad(2.3)$ & 0.004 \\
\hline RPV + TDF + FTC & $25 \quad(2.7)$ & $37 \quad(4.0)$ & 0.049 & $4 \quad(2.4)$ & $(6.1)$ & 0.053 & $2 \quad(3.6)$ & $4 \quad(7.9)$ & 0.257 \\
\hline NVP+TDF+FTC & $26 \quad(2.8)$ & $18 \quad(1.9)$ & 0.056 & $5 \quad(3.0)$ & (3.0) & 0.987 & $2 \quad(3.6)$ & $2 \quad(3.2)$ & 0.852 \\
\hline EFV+AZT + LAM & $25 \quad(2.7)$ & $9 \quad(1.0)$ & $<0.001$ & $3 \quad(1.8)$ & $(1.2)$ & 0.470 & $2 \quad(3.6)$ & $0 \quad(0.8)$ & 0.029 \\
\hline
\end{tabular}

Mean annualized health care costs per patient were 20.0\% higher for switchers than for nonswitchers ( $\$ 37,641$ vs. $\$ 31,355$; $P<0.001$; Figure 2). When total health care costs were subdivided, switchers had significantly higher costs than nonswitchers for pharmacy ( $\$ 28,287$ vs. $\$ 24,758, P<0.001)$, ambulatory $(\$ 4,993$ vs. $\$ 3,770, P=0.008$ ), and emergency ( $\$ 243$ vs. $\$ 167$, $P=0.011)$ categories. By far, the largest proportion of pharmacy costs were attributable to HIV-related medications compared with all other medication types (89\% [ $\$ 25,061 / \$ 28,287]$ for switchers, 93\% [ $\$ 22,949 / \$ 24,758]$ for nonswitchers; Figure 2).

In the virologically stable subset, follow-up annualized health care utilization per patient was significantly different for switchers versus nonswitchers only for the ambulatory category ( 14.8 visits vs. 12.3 visits, $P=0.049$; Table 3 ). Trends in follow-up annualized health care costs per patient were similar to those for the full population (Figure 2); however, only total and pharmacy costs were significantly higher for switchers versus nonswitchers $(\$ 37,120$ vs. $\$ 31,771$ for total costs, $\$ 30,724$ vs. 26,313 for pharmacy costs; $P=0.006$ for both). HIV-related medications again constituted the vast proportion of pharmacy costs compared with all other medication types; the percentage of pharmacy costs attributable to HIV-related medications was $85 \%(\$ 26,091 / \$ 30,724)$ among switchers and $92 \%(\$ 24,213 / \$ 26,313)$ for nonswitchers (Figure 2).
Among virologically+clinically stable patients, followup annualized health care utilization per patient was not significantly different between cohorts (Table 3). Only pharmacy costs were significantly higher for switchers versus nonswitchers ( $\$ 29,205$ vs. $\$ 26,351, P=0.001$; Figure 2), with HIV-related medications accounting for $96 \%(\$ 28,148 / \$ 29,205)$ and $92 \%(\$ 24,338 / \$ 26,351)$ of pharmacy costs among switchers and nonswitchers, respectively.

After adjustment for demographics, initial ART regimen, pretreatment comorbidities, and baseline health care costs, followup annualized health care costs per patient in the full population were $10.9 \%$ higher among switchers versus nonswitchers (cost ratio $[C R]=1.109,95 \%$ confidence interval $[C I]=1.058$ 1.161; Table 3). The subset analyses yielded similar results; adjusted costs for switchers versus nonswitchers were 8.9\% higher among virologically stable patients $(C R=1.089,95 \%$ $\mathrm{CI}=1.026-1.155)$ and $10.0 \%$ higher among virologically + clinically stable patients ( $\mathrm{CR}=1.100,95 \% \mathrm{CI}=1.015-1.191)$.

\section{Times to Virologic Failure and Next ART Regimen Change}

Kaplan-Meier analysis revealed that time to virologic failure was shorter among switchers than nonswitchers in the full population $(P<0.001$; Appendix B1, available in online article); however, this difference was not statistically significant in 
TABLE 3 Follow-up Annualized Health Care Utilization and Adjusted Health Care Costs Per Patient

\begin{tabular}{|c|c|c|c|c|c|c|c|c|c|}
\hline & \multicolumn{3}{|c|}{ Full Population } & \multicolumn{3}{|c|}{ Virologically Stable } & \multicolumn{3}{|c|}{ Virologically + Clinically Stable } \\
\hline & $\begin{array}{l}\text { Switchers } \\
(\mathrm{n}=927)\end{array}$ & $\begin{array}{l}\text { Nonswitchers } \\
\begin{array}{c}(\mathrm{n}=18,511 \\
\text { weighted } \\
\mathrm{n}=927)\end{array}\end{array}$ & $\begin{array}{c}95 \% \text { CI or } \\
P \text { Value }^{\mathrm{a}}\end{array}$ & $\begin{array}{l}\text { Switchers } \\
(\mathrm{n}=168)\end{array}$ & $\begin{array}{l}\text { Nonswitchers } \\
(\mathrm{n}=3,301 \\
\text { weighted } \\
\mathrm{n}=168)\end{array}$ & $\begin{array}{c}95 \% \text { CI or } \\
P \text { Value }^{\mathrm{a}}\end{array}$ & $\begin{array}{l}\text { Switchers } \\
(\mathrm{n}=55)\end{array}$ & $\begin{array}{l}\text { Nonswitchers } \\
(\mathrm{n}=1,100 \\
\text { weighted } \\
\mathrm{n}=55)\end{array}$ & $\begin{array}{c}95 \% \text { CI or } \\
P \text { Value }^{\mathrm{a}}\end{array}$ \\
\hline \multicolumn{10}{|c|}{ Health care utilization, mean (SD) ${ }^{b}$} \\
\hline Ambulatory visits & $14.7(15.6)$ & $11.6(13.6)$ & $<0.001$ & $14.8(16.4)$ & $12.3(13.7)$ & 0.049 & $12.1(11.2)$ & $12.1 \quad(13.2)$ & 0.979 \\
\hline Emergency visits & $0.9 \quad(3.4)$ & $0.6 \quad(3.3)$ & 0.027 & $0.8 \quad(2.7)$ & $0.9 \quad(4.8)$ & 0.652 & $0.5 \quad(1.4)$ & $(3.4)$ & 0.193 \\
\hline Inpatient visits & $0.1 \quad(0.6)$ & $0.1 \quad(0.6)$ & 0.120 & $0.1 \quad(0.3)$ & $0.1 \quad(0.3)$ & 0.915 & $0.1 \quad(0.3)$ & $(0.4)$ & 0.365 \\
\hline $\begin{array}{l}\text { Length of inpatient } \\
\text { visits in days }\end{array}$ & $11.2(32.7)$ & $8.7(40.5)$ & 0.485 & 7.9 (11.4) & $4.6 \quad(9.3)$ & 0.346 & $6.8 \quad(6.5)$ & $(7.5)$ & 0.366 \\
\hline Cost ratio & 1.109 & - & $1.058-1.161^{\mathrm{e}}$ & 1.089 & - & $1.026-1.155^{\mathrm{e}}$ & 1.100 & - & $1.105-1.191$ \\
\hline Adjusted costs ${ }^{c, d}$ & $\$ 36,035$ & $\$ 32,507$ & $<0.001$ & $\$ 35,779$ & $\$ 32,868$ & 0.005 & $\$ 35,297$ & $\$ 32,096$ & 0.020 \\
\hline \multicolumn{10}{|c|}{$\begin{array}{l}\text { aP values are given unless otherwise indicated. } \\
\text { bMeans and SDs are weighted and normalized to the matched switcher cohort. P values are adjusted using a robust (sandwich) variance estimate to account for correlation } \\
\text { due to multiple observations per patient. } \\
\text { cAdjusted for initial ART regimen, index year, geographic region, sex, age, Charlson comorbidity score, and baseline health care costs. } \\
\text { dObservations read =19,438 ( } 927 \text { switchers, 18,511 nonswitchers) for the full population; 3,469 (168 switchers, 3,301 nonswitchers) for the virologically stable cohort; and 1,155 } \\
\text { (55 switchers, 1,100 nonswitchers) for the virologically + clinically stable cohort. Nonswitchers were weighted to represent a single nonswitcher comparator for each switcher. } \\
\text { e95\% CI. }\end{array}$} \\
\hline
\end{tabular}

either of the virologically stable subsets (Appendix B3 and $\mathrm{B} 5$, respectively; $P>0.05$ for both). Time to next ART regimen change was significantly shorter among switchers than nonswitchers in the full population and in the virologically stable and virologically + clinically stable subsets (Appendix B2, B4, and $\mathrm{B} 6$, respectively; $P<0.001$ for all).

\section{Discussion}

This real-world analysis was designed to assess the health care burden associated with changes in ART therapy among HIV-positive patients who had longevity on their first-line regimens. Among virologically stable patients, those who switched therapies had higher total health care costs compared with nonswitchers; this difference remained significant after adjustment for potential confounders. The observed cost increase was driven primarily by higher pharmacy costs, which were dominated by HIV-related medication costs. These findings support the hypothesis that ART switching among stable patients with HIV may not confer economic benefit.

Although most baseline characteristics were similar between study cohorts, comorbidity burden was higher among switchers in the full population. Furthermore, over $43 \%$ of the 297 patients with available HIV RNA test results were found to have detectable viral loads. These findings suggest that results of the full-population analysis were likely confounded by the presence of patients with overall poorer health or lack of viral suppression, so analysis of the virologically stable subsets with more similar comorbidity burden between cohorts is more clinically relevant. Follow-up unadjusted annualized health care costs among ART switchers versus nonswitchers were 16.8\% higher in the virologically stable subset $(\$ 37,120$ vs. $\$ 31,771)$ but not significantly different in the virologically + clinically stable subset. After adjustment for ART regimen, demographic data, comorbidities, and baseline total health care costs, followup costs were significantly higher among switchers: $8.9 \%$ higher in the virologically stable subset and $10.0 \%$ higher in the virologically+clinically stable subset $(\$ 35,779$ vs. $\$ 32,868$ and $\$ 35,297$ vs. $\$ 32,096$, respectively).

While peer-reviewed studies of the economic effect of ART switching are extremely limited-particularly regarding claims database analyses-our results are comparable to those of Solem et al. (2014), who found that covariate-adjusted annualized health care costs for patients with HIV were $\$ 28,861$ for the initial treatment regimen and $\$ 35,805$ after a switch to second-line therapy. ${ }^{19}$ This $24 \%$ increase was much steeper than that observed in our study; however, viral load data were not available for the Solem et al. sample, and patients were required to have been on their initial regimens for only 90 days (vs. 6 months in our study). The Solem et al. analysis thus may have contained a higher proportion of patients with poorly controlled disease compared with our virally suppressed subsets, which could substantially increase costs.

In contrast to our results, Llibre et al. (2013) found that ART switching among virally suppressed patients resulted in significant cost savings while maintaining virologic control in about $98 \%$ of patients. ${ }^{20}$ However, their study was undertaken specifically to assess the effect of cost reduction measures instituted in one Spanish hospital. Not only were switches to more expensive therapies excluded from the analysis, but therapy adjustments were made by a collaborative medical team that 


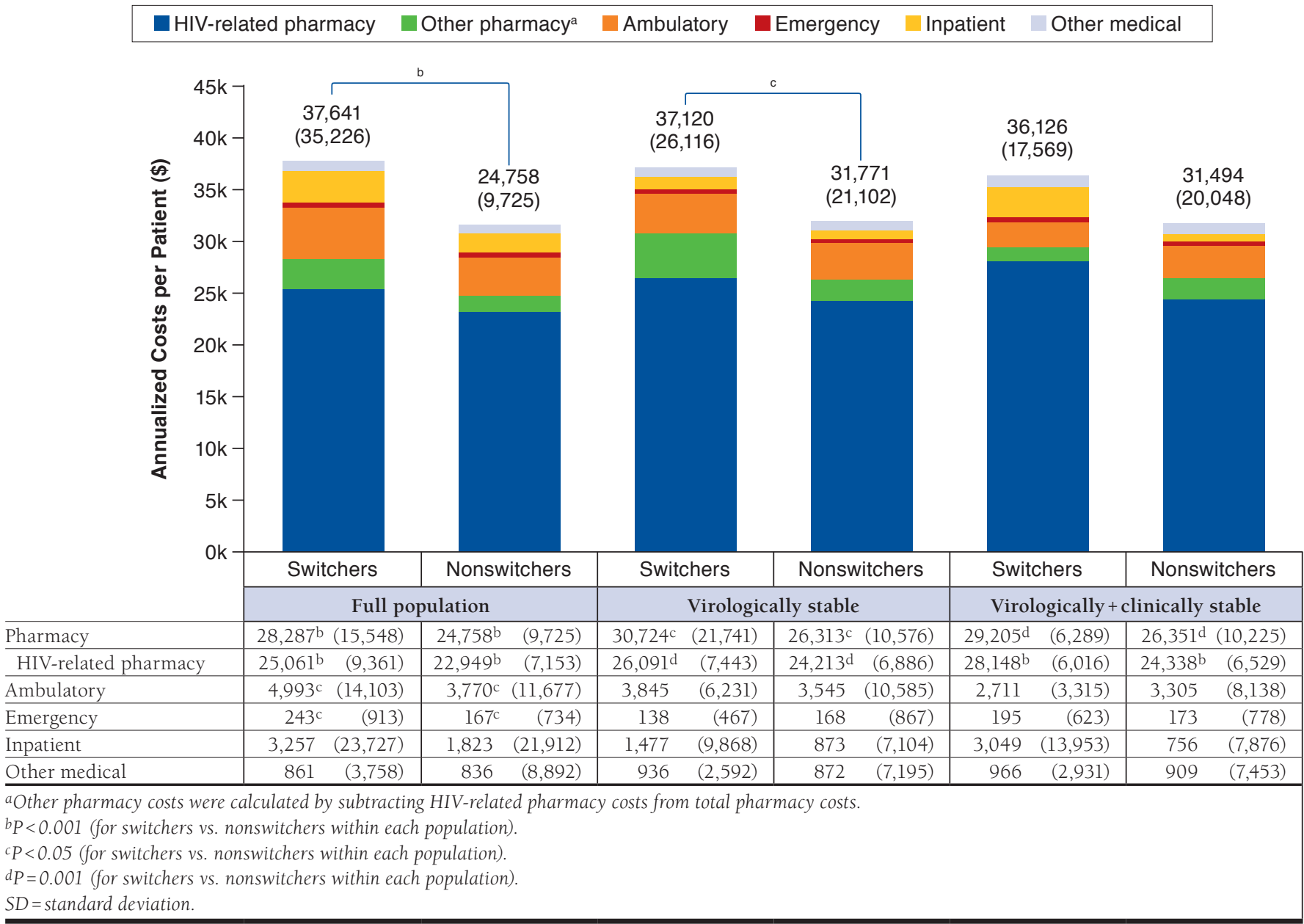

was well apprised of the program goals and whose decisions were bound by criteria established to maintain treatment safety and efficacy. Our study, on the other hand, used claims data from large numbers of patients visiting different health care providers across the United States, so definitive reasons for therapy changes were unknown and any number of factors may have come into play: cost, tolerance, convenience, adherence, or even the simple allure of a novel drug's arrival on the market. Although Llibre et al. showed that ART switching can be cost-effective, the disparity between their results and ours underscores the careful consideration with which therapy changes must be undertaken in order to derive clinically and economically beneficial outcomes.

Most studies of HIV therapy economics conclude that pharmacy costs represent the largest share of health care costs for patients with HIV, and our study is no exception. ${ }^{19,25,26}$ Pharmacy costs among virologically stable patients were significantly higher for switchers, constituting $84 \%$ of total health care costs for switchers and $81 \%$ for nonswitchers. Importantly, the vast majority of these costs were attributable to HIV-related medications, with HIV-related pharmacy costs significantly higher for switchers versus nonswitchers in all cohorts. Although the number of ambulatory visits was slightly higher for switchers versus nonswitchers in the virologically stable subset, ambulatory costs were not significantly different between cohorts in either of the subset analyses. In a previous report, Martin et al. (2007) suggested that incremental costs associated with the number of ART switches were predominantly due to the occurrence of side effects and other health care-related costs. ${ }^{25}$ However, considering that we observed no significant switching-associated cost increases in nonpharmacy categories among virologically stable patients, this does not appear to have been a major factor in our study. Taken together, our findings suggest that increased health care 
costs for stable switchers are caused primarily by the use of more expensive drugs, prescription overlap, or other phenomena related to the switch itself (e.g., requisite office visits or laboratory tests). Moreover, these findings raise the question of what, if any, benefit was derived from ART changes in this study-particularly among patients with no apparent clinical reason for switching. Further research assessing post-switch comorbidity burden and ART treatment patterns, which were not analyzed in this study, may help verify the reasons for therapy changes and subsequent increases in pharmacy costs.

Exploratory analyses revealed that among switchers in the virologically stable and virologically + clinically stable subsets, time to next ART regimen change was significantly shorter compared with nonswitchers. Although this could suggest decreased stability of virologic suppression among switchers, we cannot discount the possibility that patients who had already switched regimens may have been more open to considering a subsequent switch.

The dramatic expansion of ART options over the years has made it possible to achieve and maintain virologic suppression in more patients than ever before; nevertheless, switching therapies can lead to unanticipated outcomes such as new drug tolerability issues, drug interactions, or loss of virologic control. ${ }^{14,15,27,28}$ In one study, patients with HIV who switched to a second-line therapy while virally suppressed were nearly 3 times more likely than nonswitchers to suffer subsequent virologic failure. ${ }^{28}$ Given these possibilities, decision making in clinical practice should be based on solid evidence from clinical trials. ${ }^{6}$ However, because the number of patients who experience virologic failure has fallen drastically thanks to improvements in drug efficacy, ART switching trials are often conducted among virologically stable patients, with an endpoint of virologic noninferiority (typically defined as 95\% confidence that the tested drug is at least $88 \%-90 \%$ as effective as the comparator). ${ }^{27}$ Some regimens may thus be less effective in the real world than they are in clinical trials. ${ }^{27}$ This observation, in conjunction with our findings that switching from the first-line ART regimen is associated with increased health care costs (and possibly with shorter time to virologic failure) among virally suppressed patients, bolsters the argument that ART changes should not be considered without a clear clinical reason.

Treating HIV is expensive, and ART medications account for a substantial proportion of HIV-related medical costs. ${ }^{19,25,26}$ Although maintenance of virologic suppression with minimal adverse effects is of paramount importance in determining a therapeutic strategy for treating patients with HIV, the parallel necessity of cost management is becoming increasingly evident, particularly in light of the ballooning economic burden of health care in the United States. Our findings suggest that switching ART in stable patients only when there is clear clinical need may help curtail unnecessary expense-a critical consideration as the lifetime costs of HIV treatment continue to rise with the aging of the HIV-positive population. ${ }^{29}$

\section{Limitations}

Our findings should be interpreted in light of certain limitations inherent to claims-based analyses. Because this study was conducted in a large U.S. managed care population among a subset of patients meeting the inclusion criteria, the results may not be generalizable to other populations (e.g., patients who are uninsured or share other similarities with those excluded from the analysis). The presence of a diagnosis code on a medical claim does not prove the presence of disease, since diagnoses may be coded incorrectly or included as ruleout criteria. Furthermore, pharmacy claims do not indicate whether medication was taken as prescribed, and medications provided as samples by a health care provider or as part of a clinical trial are not accounted for in claims data.

Identification of stable patients in this study could only be inferred from the information available in the administrative claims and linked laboratory results. Although the requirement that patients be treated with their initial ART regimen for at least 6 months was intended to increase the proportion with no clinical reason to switch therapies, we found that a substantial number of switchers in the full population had detectable viral loads, which reduced the clinical relevance of the full-population analysis. For the subanalyses of virologically suppressed patients, only those with available lab results were considered. This sample size limitation may have resulted in insufficient power to detect significant results; moreover, the comparability of patients with and without available virologic test results is not known. Finally, some patients in the virologically + clinical stable subset may have been misclassified if their clinical reasons for switching therapies were unobservable in the claims data.

\section{Conclusions}

Patients with HIV who switched regimens after having been stable on their first-line ART had significantly higher downstream costs than those who did not switch. The observed health care cost increases were driven mostly by higher HIV-related pharmacy costs. Our findings suggest that ART switches among stable patients may be costly and should be undertaken only when clinically warranted.

\section{Authors}

LISA ROSENBLATT, MD, MPH, and ANGELINA VILLASISKEEVER, MD, MSc, Bristol-Myers Squibb, Plainsboro, New Jersey. AMI R. BUIKEMA, MPH; JERRY SEARE, MD; LINDSAY G. S. BENGTSON, PhD, MPH; JONATHAN JOHNSON, MS; and FENG CAO, PhD, Optum, Eden Prairie, Minnesota.

AUTHOR CORRESPONDENCE: Ami R. Buikema, MPH, Optum, 11000 Optum Circle, MN101-300, Eden Prairie, MN 55344.

Tel.: 952.205.7725; E-mail: ami.buikema@optum.com. 


\section{DISCLOSURES}

This work was funded by Bristol-Myers Squibb (BMS), which participated in the design of the study, interpretation of the data, revision of the manuscript, and the decision to submit the manuscript for publication. Rosenblatt is an employee and stock owner of BMS; Villasis-Keever was an employee of BMS at the time this study was conducted and is currently an employee of Janssen. Buikema is an employee and stock owner of Optum, and Seare, Bengston, Johnson, and Cao are employees of Optum, which was contracted by BMS to conduct the study. Optum contracts with pharmaceutical companies, such as Janssen, Merck, EMD Serano, GlaxoSmithKline, and Gilead, to conduct research in HIV. Optum is also a subsidiary of a health plan that has interest in managing the health and associated costs of patients with HIV.

Study concept and design were contributed by Rosenblatt and Buikema, along with the other authors. Cao and Johnson took the lead in data collection, along with Buikema, Seare, and Bengston. Data interpretation was performed by Buikema, Seare, Bengston, and Villasis-Keever. The manuscript was written by Buikema and Bengston, along with Rosenblatt, Seare, Johnson and Villasis-Keever, and revised by Rosenblatt, Villasis-Keever, and Johnson, along with the other authors.

\section{ACKNOWLEDGMENTS}

Medical writing services were provided by Yvette M. Edmonds, PhD; programming assistance was provided by Lynn Wacha; and statistical consultation was provided by Lee Brekke, PhD (all employed by Optum).

\section{REFERENCES}

1. Samji H, Cescon A, Hogg RS, et al. Closing the gap: increases in life expectancy among treated HIV-positive individuals in the United States and Canada. PLoS One. 2013;8(12):e81355.

2. Panel on Antiretroviral Guidelines for Adults and Adolescents. Guidelines for the use of antiretroviral agents in HIV-1-infected adults and adolescents. 2016. Available at: https://aidsinfo.nih.gov/guidelines/html/1/adult-andadolescent-arv-guidelines/0. Accessed May 6, 2017.

3. Stone VE, Jordan J, Tolson J, Miller R, Pilon T. Perspectives on adherence and simplicity for HIV-infected patients on antiretroviral therapy: self-report of the relative importance of multiple attributes of highly active antiretroviral therapy (HAART) regimens in predicting adherence. J Acquir Immune Defic Syndr. 2004:36(3):808-16.

4. Glass TR, De Geest S, Weber R, et al. Correlates of self-reported nonadherence to antiretroviral therapy in HIV-infected patients: the Swiss HIV Cohort Study. J Acquir Immune Defic Syndr. 2006;41(3):385-92.

5. Paterson DL, Swindells S, Mohr J, et al. Adherence to protease inhibitor therapy and outcomes in patients with HIV infection. Ann Intern Med. 2000;133(1):21-30

6. Taiwo B, Murphy RL, Katlama C. Novel antiretroviral combinations in treatment-experienced patients with HIV infection: rationale and results. Drugs. 2010;70(13):1629-42.

7. Drechsler H, Powderly WG. Switching effective antiretroviral therapy: a review. Clin Infect Dis. 2002;35(10):1219-30.

8. Brunetta J, Moreno Guillen S, Antinori A, et al. Patient-reported outcomes after a switch to a single-tablet regimen of rilpivirine, emtricitabine and tenofovir DF in HIV-l-positive, virologically suppressed individuals: additional findings from a randomized, open-label, 48-week trial. Patient. 2015;8(3):257-67.

9. Campo RE, Cohen C, Grimm K, Shangguan T, Maa J, Seekins D. Switch from protease inhibitor- to efavirenz-based antiretroviral therapy improves quality of life, treatment satisfaction and adherence with low rates of virological failure in virologically suppressed patients. Int J STD AIDS. 2010;21(3):166-171.

10. Potard V, Chassany O, Lavignon M, Costagliola D, Spire B. Better healthrelated quality of life after switching from a virologically effective regimen to a regimen containing efavirenz or nevirapine. AIDS Care. 2010;22(1):54-61.
11. Perez-Hernandez IA, Palacios R, Mayorga M, et al. Lipid changes in HIV-patients switching to the coformulated single tablet FTC/RPV/TDF (Eviplera). Efficacy and safety analysis. GeSida Study 8114. J Int AIDS Soc 2014;17(4 Suppl 3):19795.

12. Airoldi M, Zaccarelli M, Bisi L, et al. One-pill once-a-day HAART: a simplification strategy that improves adherence and quality of life of HIVinfected subjects. Patient Prefer Adherence. 2010;4:115-125.

13. Fabbiani M, Zaccarelli M, Grima P, et al. Single tablet regimens are associated with reduced Efavirenz withdrawal in antiretroviral therapy naive or switching for simplification HIV-infected patients. BMC Infect Dis. 2014;14:26.

14. Martinez E, Arranz JA, Podzamczer D, et al. A simplification trial switching from nucleoside reverse transcriptase inhibitors to once-daily fixed-dose abacavir/lamivudine or tenofovir/emtricitabine in HIV-1infected patients with virological suppression. J Acquir Immune Defic Syndr. 2009;51(3):290-97.

15. Eron JJ, Young B, Cooper DA, et al. Switch to a raltegravir-based regimen versus continuation of a lopinavir-ritonavir-based regimen in stable HIV-infected patients with suppressed viraemia (SWITCHMRK 1 and 2): two multicentre, double-blind, randomised controlled trials. Lancet. 2010;375(9712):396-407

16. Arribas JR, Delgado R, Arranz A, et al. Lopinavir-ritonavir monotherapy versus lopinavir-ritonavir and 2 nucleosides for maintenance therapy of HIV: 96-week analysis. J Acquir Immune Defic Syndr. 2009;51(2):147-52.

17. Arribas JR, Horban A, Gerstoft J, et al. The MONET trial: darunavir/ ritonavir with or without nucleoside analogues, for patients with HIV RNA below 50 copies/ml. AIDS. 2010;24(2):223-30.

18. Meenan RT, O'Keeffe Rosetti M, Kimes T, et al. Incidence-based costs of multiple HAART switches among HIV-infected patients in an HMO. Clin Med Res. 2010;8(1):52

19. Solem CT, Snedecor SJ, Khachatryan A, et al. Cost of treatment in a US commercially insured, HIV-l-infected population. PLoS One. 2014;9(5):e98152

20. Llibre JM, Cardona G, Santos JR, et al. Antiretroviral treatment switch strategies for lowering the costs of antiretroviral therapy in subjects with suppressed HIV-1 viremia in Spain. Clinicoecon Outcomes Res. 2013;5:215-21.

21. Quan H, Li B, Couris CM, et al. Updating and validating the Charlson comorbidity index and score for risk adjustment in hospital discharge abstracts using data from 6 countries. Am J Epidemiol. 2011;173(6):676-82.

22. Healthcare Cost and Utilization Project (HCUP). Clinicial Classifications Software for ICD-9-CM. Agency for Healthcare Research and Quality. 2015. Available at: https://www.hcup-us.ahrq.gov/toolssoftware/ccs/ccs.jsp. Accessed May 6, 2017.

23. American Hospital Formulary Service. AHFS pharmacologic-therapeutic classification. 2015. Available at: http://www.ahfsdruginformation.com/ahfspharmacologic-therapeutic-classification/. Accessed May 6, 2017.

24. U.S. Bureau of Labor Statistics. Consumer Price Index. Medical care. Series ID: SUUR0000SAM. 2012. Available at: http://data.bls.gov/cgi-bin/ surveymost?su. Accessed May 6, 2017.

25. Martin S, Foley K, Baser O. Incremental medical costs associated with increased changes in HAART regimens in a U.S. patient sample. Presented at: 4th IAS Conference on HIV Pathogenesis, Treatment and Prevention; July 22-25, 2007; Sydney, Australia. Abstract WEPEB047.

26. Gebo KA, Fleishman JA, Conviser R, et al. Contemporary costs of HIV healthcare in the HAART era. AIDS. 2010;24(17):2705-15.

27. Carr A, Hoy J, Pozniak A. The ethics of switch/simplify in antiretroviral trials: non-inferior or just inferior? PLoS Med. 2012;9(7):e1001240.

28. Hull M, Cescon A, Raboud J, et al. Switching from first antiretroviral therapy regimen while virologically suppressed is associated with increased risk of subsequent virologic failure. Presented at: 20th International AIDS Conference; July 20-25, 2014; Melbourne, Australia. Available at: http://pag. aids2014.org/abstracts.aspx?aid=4291. Accessed May 6, 2017.

29. Krentz HB, Gill MJ. Increased costs of HIV care associated with aging in an HIV-infected population. HIV Med. 2015;16(1):38-47. 


\section{APPENDIX A Guidance for Categorizing Switchers as Unstable}

\begin{tabular}{|c|c|c|}
\hline Switch From & Switch To & Clinical Criteria for "Unstable" Categorization \\
\hline $\mathrm{TDF}+\mathrm{FTC}$ & $\mathrm{ABC}+3 \mathrm{TC}$ & $\begin{array}{l}\text { Poor renal function (GFR }<60 \text {, renal secondary hypertension, chronic kidney } \\
\text { disease, renal failure, Fanconi syndrome); bone fracture; osteoporosis }\end{array}$ \\
\hline $\mathrm{ABC}+3 \mathrm{TC}$ & TDF +FTC & Heart disease (coronary artery disease, congestive heart failure) \\
\hline PI & Non-PI & $\begin{array}{l}\text { Hypercholesterolemia, nephrolithiasis, kidney dysfunction (except when switching } \\
\text { from DRV), jaundice (when switching from ATV only), testicular hypofunction, } \\
\text { abnormal blood glucose }\end{array}$ \\
\hline ATV & Non-PI & $\begin{array}{l}\text { Hyperbilirubinemia, if coupled with clinical diagnosis indicating jaundice, } \\
\text { icterus, etc. }\end{array}$ \\
\hline EFV & Integrase, RPV & Dyslipidemia \\
\hline EFV & Integrase, RPV, PI & Anxiety, depression, sleep disturbance, migraine \\
\hline NVP & Any & Abnormal liver function test, hepatitis \\
\hline EFV or boosted PI & $\begin{array}{l}\text { RAL, DTG, RPV, NVP, ATV } \\
\text { (unboosted) }\end{array}$ & Hypercholesterolemia \\
\hline Any & $\begin{array}{l}\text { MVC, T-20, nucleotide/ } \\
\text { nucleoside-sparing, or other } \\
\text { nonstandard regimen }\end{array}$ & Any \\
\hline Trizivir & $\begin{array}{l}\text { Standard regimen (anchor }+2 \\
\text { backbone) }\end{array}$ & Switch likely recommended to choose better therapy options and eliminate ZDV \\
\hline ZDV & $\begin{array}{l}\text { Standard regimen (anchor }+2 \\
\text { backbone) }\end{array}$ & Switch likely recommended to eliminate ZDV \\
\hline
\end{tabular}


APPENDIX B Kaplan-Meier Analyses of Time to Virologic Failure and Time to Next ART Regimen Change

1. Time to Virologic Failure: Full Population

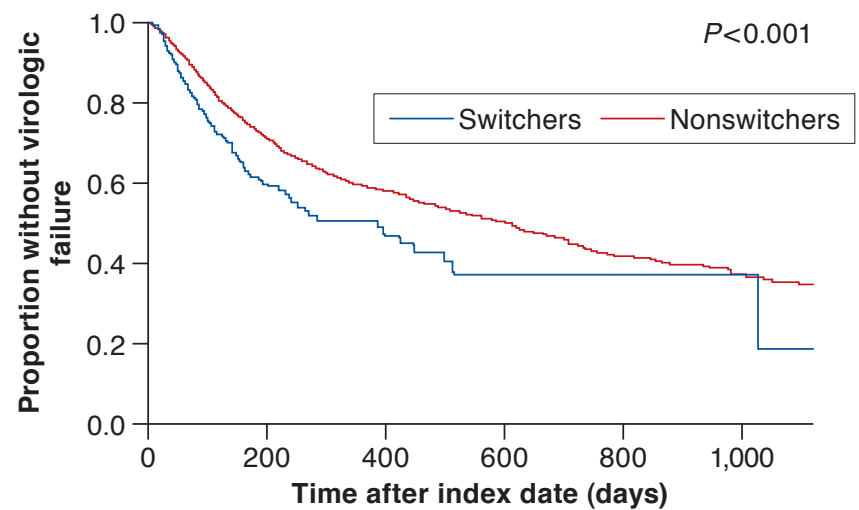

3. Time to Virologic Failure: Virologically Stable

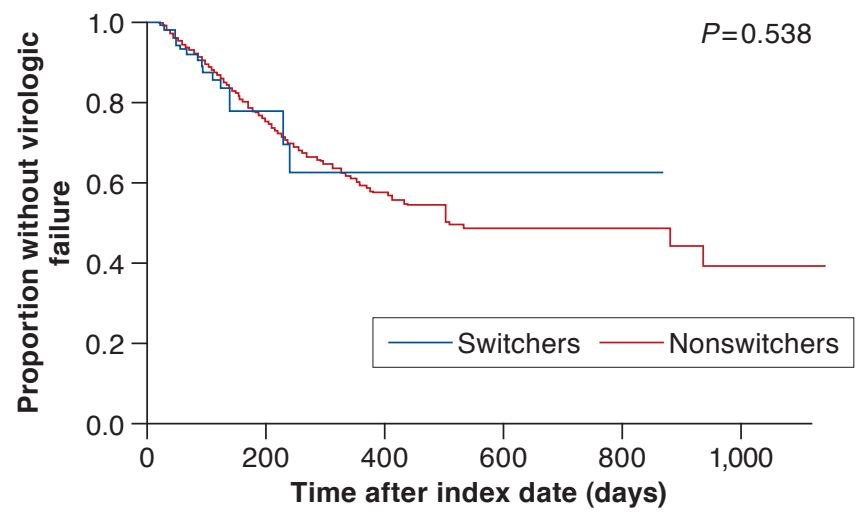

5. Time to Virologic Failure: Virologically +Clinically Stable

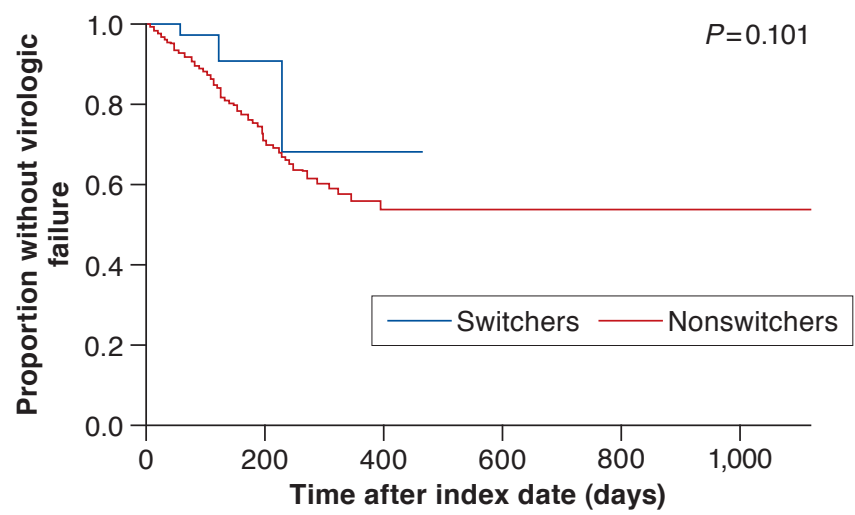

2. Time to Next ART Regimen Change: Full Population

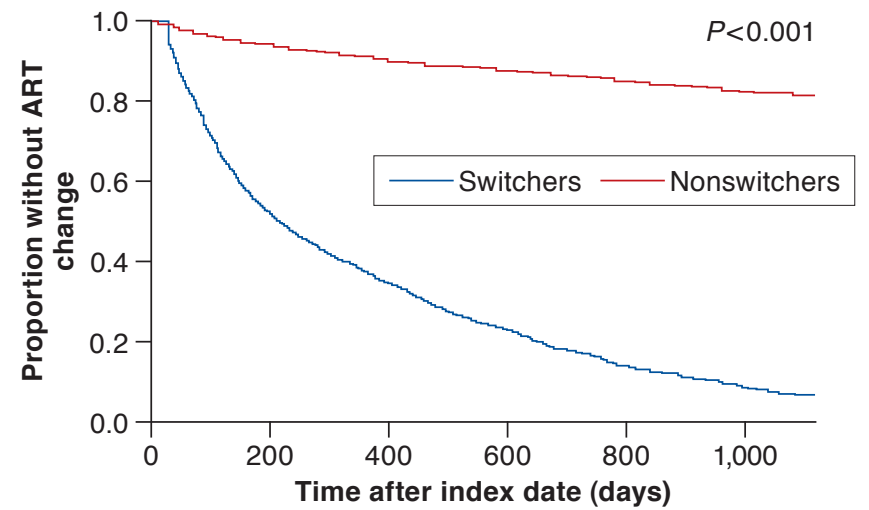

4. Time to Next ART Regimen Change: Virologically Stable

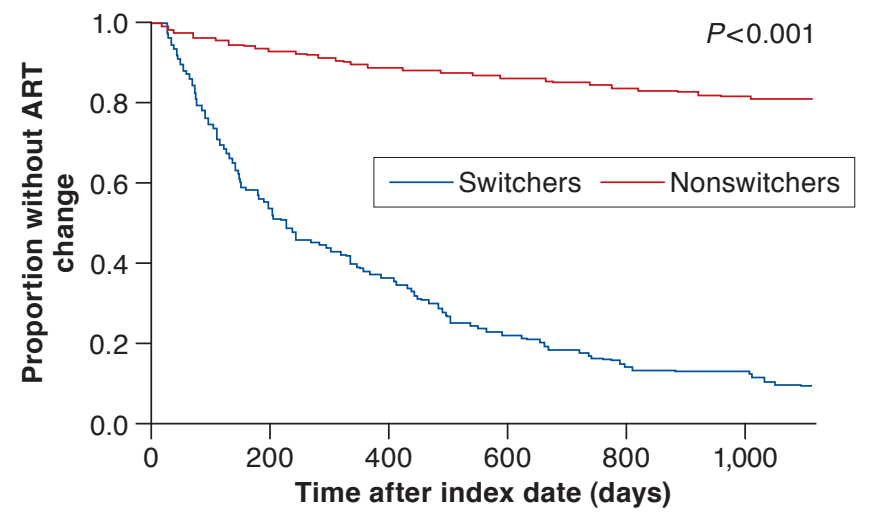

6. Time to Next ART Regimen Change: Virologically+Clinically Stable

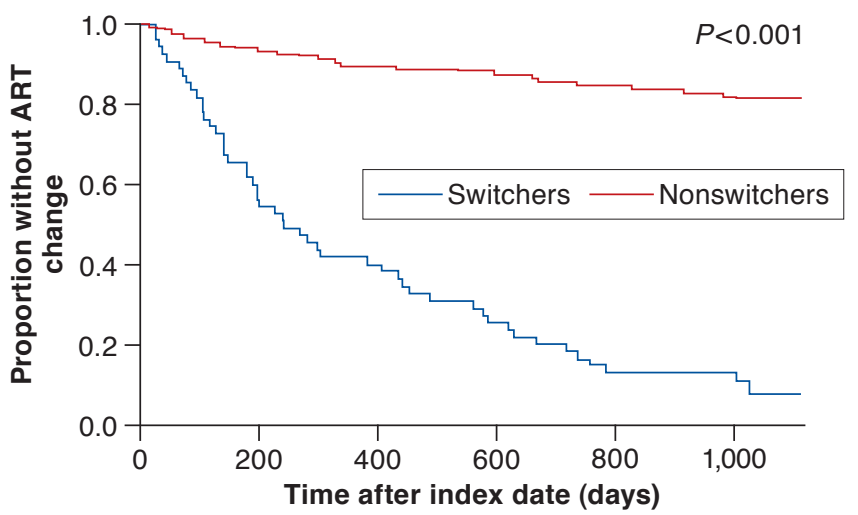

Note: For graphs 2, 4, and 6, data were censored at the end of the observation period for patients without a second regimen change. Because matching was conducted with replacement, patients in the nonswitcher cohort who subsequently changed ART regimens could have become switchers (with their own matched comparators). $A R T=$ antiretroviral therapy. 